\title{
Assessment of Agreement between Gated SPECT Myocardial Perfusion Imaging and Gated SPECT Blood Pool Imaging for Measurement of Left Ventricular Ejection Fraction in Coronary Artery Disease
}

\author{
1.Azmal K Sarker, ${ }^{1}$ Raihan Hussain, ${ }^{1}$ Faria Nasreen, ${ }^{1}$ Lutfun Nisa, ${ }^{2}$ Monsurul Hoo, ${ }^{1}$ Khokon K Nath, ${ }^{1}$ Nurjahan Khatun, \\ 1 Saiful Islam and 3 Faridul Alam \\ ${ }^{1}$ National Institute of Nuclear Medicine and Allied Sciences, BAEC, Bangabandhu Sheikh Mujib Medical University campus, Shahbag, Dhaka \\ ${ }^{2}$ Mürdoch Children's Research Institute, Melbourne, Australia \\ ${ }^{3}$ Bangladesh Institute of Health Scienees, Dhaka
}

Correspondence Address: Dr, Azmal K Sarker, Assistant Professor, National Institute of Nuclear Medicine and Allied Sciences, Dhaka, e-mailyaksninmas@gmailicom

\begin{abstract}
Objective: Quantitative assessment of left ventricular ejection fraction (LVEF) from radionuclide cardiac imaging study has both diagnostic and prognostic value in coronary artery disease (CAD), Gated SPECT blood pool imaging (GSBPI) and gated SPECT myocardial perfusion imaging (GSMPI) are two technically comparable radionuclide methods for non-invasive measurement of LVEF. While the former is a gold standard the latter is popular as it provides a wider array of information. This study was carried out to bridge the lack in the existing body of evidence regarding assessment of agreement of between GSBPI and GSMPI for measurement of LVEF in CAD. The objective of this study was to validate the LVEF measurements from routine GSMPI as a valuable parameter for clinical decision making through assessment of agreement between GSMPI and GSBPI performed in a short interval, in same patient having $C A D$.
\end{abstract}

Patients and Methods: A total of 28 patients (threc female) was observed with a mean age of $54.2+8.5$ years during February to May 2012. All patients underwent GSBPI and GSMPI with a gap of three to seven days in between. LVEF measured by GSBPI performed at rest was compared with LVEF measured in rest phase of onc day stress-rest GSMPI. Agreement analysis was done with Bland Altman plot:

Results; Mean LVEF measurements show an apparent overall slight underestimation by GSBPI $(54.8 \pm 25.3)$ in comparison to GSMPI (56.9 \pm 25). Bland Altman plots show that the differenees between GSBPI and GSMPI for measurement of LVEF at rest in same patient fall within two SD of the mean difference. This finding remained similar while further categorization of study patients was done on basis of ranges of LVEF, end diastolic volume (EDV), end systolic volume (ESV), infaret size and regional wall motion abnormality (RWMA).
Conclusions: There was overall significant agreement between GSMPI and GSBPI for measurement of LVEF in CAD in this small study. This agreement remains significant irrespective of ranges of LVEF, EDV, ESV, infarct size and RWMA.

Kej Words: LVEF, Agreement, Correlation, Gated SPECT, myocardial perfusion, blood pool imaging.

\section{INTRODUCTION}

The coronary artery disease (CAD) has been evolving as a major contributor to mortality and disability in Bangladesh $(1,2,3)$. Risk stratification in CAD is based on estimates of myocardial ischemia (4) and left ventricular ejection fraction (LVEF) $(5,6)$. Quantitative assessment of the LVEF through its diagnostic and prognostic value has been guiding clinicians and surgeons to device medical and interventional therapies in patients with CAD. An accurately estimated LVEF is therefore a crucial expectation from a non-invasive radionuclide cardiac imaging study.

Equilibrium radionuclide ventriculography (ERNV), a planar technique, since its inception in 1970 has gained reputation of being a gold standard technique for measurement of LVEF (7). It was in early eighties when nuclear cardiology emerged as a partner in CAD management in Bangladesh with the advent of multi gated radionuclide ventriculography (MUGA), a planar variety of ERNV. 
Electrocardiogram (ECG) gated SPECT blood pool imaging (GSBPI) being a tomographic variety of ERNV is technically superior than planer techniques for estimation of LVEF, EDV, ESV and LV regional wall motions (8). ECG gated SPECT myocardial perfusion imaging (GSMPI) provides quantifications of LVEF, LV cavity end systolic volume (ESV), end diastolic volume (EDV), regional myocardial perfusion, regional wall motion and regional wall thickening. GSMPI has become a popular technique in the management of CAD in Bangladesh since 2001(9).

There are reports of validation of GSMPI against planer and SPECT blood pool techniques with good inter method correlation for measurements of LVEF and LV volumes (10). However, interchangeable use of these two methods are either opposed or kept conditional by a number of recommendations. Sole reliance on correlation coefficient is not appropriate for analysis of measurement method comparison (11). A high correlation can falsely come out of a data containing wide range of value and can conceal considerable lack in agreement. Existing literatures lack study regarding assessment of agreement of LVEF measurement by two tomographic radionuclide techniques, GSMPI and GSBPI. Thus an assessment of agreement between GSMPI and GSBPI for measurement of LVEF in CAD appeared to have important clinical value. The objective of this study was to validate the LVEF measurements from routine GSMPI as a valuable parameter for clinical decision making through assessment of agreement between GSMPI and GSBPI performed in a short interval, in same patient having $\mathrm{CAD}$.

\section{PATIENTS AND METHODS}

A total of 27 adult male and female patients were included by non-random sampling technique who were referred either for screening of suspected $\mathrm{CAD}$ or for clinical evaluation of known CAD under medical treatment or post coronary revascularization. All patients underwent GSMPI and GSBPI within a span of three to seven days. No patient has any intervening cardiac event. Left ventricular parameters were measured in rest phase of routine one day standard stress-rest GSMPI and compared against those obtained from GSBPI performed at rest on a separate day.

This cross sectional analytical study was carried out over a period of one year from July 2011 to June 2012 at the former Institute of Nuclear Medicine \& Ultrasound (INM \& U), which is currently known as National Institute of Nuclear Medicine \& Allied Sciences (NINMAS). The study population consisted of patients referred to the institute for GSMPI. The sample size was calculated for discordance rate $(\alpha)$ of 0.10 and tolerance probability ( $\beta$ ) of $95 \%$ for agreement of two measurement methods assuming no discordant pair of measurement $(\mathrm{k}=0)$ allowed (12). The academic Committee of INM \& U approved the study protocol. Informed written consent was obtained from all patients. For the pre procedure counseling and evaluation of patients there was a face to face interview session between each patient and a nuclear medicine physician. Best possible attempts were made to maintain adherence to quality control recommendations from renowned authorities $(10,13)$ at all levels of patient preparation, radiopharmaceutical preparation and dispensing, image acquisition, image processing and image interpretation. Image acquisition for both the GSMPI and GSBPI were conducted with Siemens E cam dual head gamma camera.

SPECT acquisition with ECG gating at rest phase was done 45-60 minutes after rest injection of $25 \mathrm{mCi}$ of Tc-99m-sestamibi on the same day following the post-stress scan (done 15-30 minutes after stress injection of $10 \mathrm{mCi}$ of tracer). Both the detector heads were placed at $76^{\circ}$ to each other. A zoom of 1.45 was used. A symmetric $15 \%$ energy window around the $140 \mathrm{keV} 99 \mathrm{mTc}$ photo-peak was set. Data was stored 
in 64 x 64 matrices (pixel size $6.59 \mathrm{cmm}$ and 21-27 slices in short axis). ECG gating was done with three limb leads and acquisition was set to eight frames per R-R interval (about 153ms/frame). Framing method configuration was with $60 \%$ width and forward backwards by thirds. The auto-centre set to average of 10 beats with pick bin of 10 bits and auto-tracking on. Reject PVC (Premature Ventricular Contraction) beat mode was set with PVC threshold of $300 \mathrm{msec}$. Rotation of camera heads were set to counter clock wise with starting angle at $52^{\circ}$. The detectors took 32 views over $104^{\circ}$ of rotation in a non circular orbit using a step-and-shoot method, progressing from $45^{\circ}$ right anterior oblique to $45^{\circ}$ left posterior oblique projections. During acquisition the patient was in supine position with 'head out' orientation. Acquisition time was 20 seconds per projection (about $450-750 \mathrm{k}$ counts/detector/view) requiring about 14 minutes per patient per scan. Butterworth filtering with cutoff of $0.5 \mathrm{cycles} / \mathrm{sec}$ and order 7 was used.

In vivo method for labeling of stannous pyrophosphate with Tc-99m pertechnetate was used. After 20 minutes of intravenous injection of 15-20 mg pyrophosphate, 15-20 milli Curie of Tc-99m-pertechnatate was injected in a different vein of contra-lateral arm. Immediately thereafter gated blood pool SPECT images was acquired. Standard protocol was followed for image acquisition which was same in all aspects with the GSMPI acquisition protocol as mentioned earlier, except in the frame rate with ECG gating. For three limb leads ECG gating the acquisition was set to 16 frames per R-R interval that took about $45-48$ millisecond/frame and 300-350 kilo counts/detector/view and the time of scan took 12 minute per patient per scan.

The measurements of LV volumes and EF were generated by software after manual processing of raw data had been done at a dedicated work station (Siemens e.soft). Quantitative assessment of SPECT perfusion was done by 4D-MSPECT v4.2 software
(Invia, LLC 2007). SPECT blood pool data was quantified with QBS 2007 (Quantitative Blood-pool SPECT, Cedars cardiac quantification software 6.5.9.1 Cedars Sinai Medical Centre, Los Angeles, California).

Statistical analysis of data was done using IBM SPSS statistics (Statistical Package for Social Sciences) version 20 (IBM corporation 2011) for windows. Descriptive statistics viz. frequency, percentage, mean, SD, range were calculated for the basic demographic characteristics and LVEF of the study patients. For analysis of agreement between GSMPI and GSBPI derived measurements of LVEF at rest, Bland-Altman plots were constructed to observe if the differences of LVEF plotted against respective geometric means of LVEF falls within two standard deviations (SD) of difference.

In this study, in addition to an overall agreement analysis, further assessment of agreement were done in the study patients among different ranges of LVEF, EDV, ESV, infarct size and wall motion. Cut offs for LVEF ranges and cut offs for EDV and ESV limits for SPECT measurements were adopted from available published literature (14-21). LVEF was categorized as 'less than $35 \%$ ', ' 35 to less than $55 \%$ ' and ' $55 \%$ or greater'. EDV was categorized as below $85 \mathrm{ml}, 85$ to $140 \mathrm{ml}$ and above $140 \mathrm{ml}$. ESV was categorized as below $15 \mathrm{ml}, 15$ to $60 \mathrm{ml}$ and above $60 \mathrm{ml}$. While assessing agreement in absence or presence of infarct, quantitative estimation of fixed defect on $17 \mathrm{LV}$ segments were assigned in to qualitative categories. For qualitative infarct size assumption, fixed defect involving up to five out of $17 \mathrm{LV}$ myocardial segments was considered as small LV infarct. Fixed perfusion defect size of more than five segments was considered as large infarct. For assessment of agreement in absence or presence of regional wall motion abnormality (RWMA), summed motion score (SMS) at rest obtained from GSMPI images were qualitatively assigned into three categories. A SMS at rest of up to 
10 were considered as normal if there was no RWMA that is visually detectable in cine views of $4 \mathrm{D}$ reconstruction images. A resting SMS of 10 to 25 was considered as mild to moderate RWMA while SMS at rest more than 25 was considered as gross RWMA.

\section{RESULTS}

Among the 27 study patients there was 24 males $(88.9 \%)$ and 3 females $(11.1 \%)$. The age range of the patients were 32 to 68 years, with a mean \pm SD of $54.19 \pm 6.15$ years. The reasons for referral for GSMPI was evaluation of myocardial viability after old myocardial infarction in 15 , assessment post coronary revascularization in four and evaluation of chest pain in eight patients (Table 1).

Table 1: Demographic characteristics of study patients

\begin{tabular}{llll}
\hline Dcmographic charactcristics & Malc & Fcmalc & All paticnts \\
\hline Age(Mean \pm SD) & $53.5 \pm 8.6$ & $59.3 \pm 7.5$ & $54.2 \pm 8.5$ \\
Weight (Mean \pm SD) & $64.9 \pm 6.9$ & $70 \pm 10$ & $65.5 \pm 7.2$ \\
Height(Mean \pm SD) & $163.3 \pm 3.3$ & $162 \pm 3.8$ & $163.2 \pm 3.3$ \\
BMI(Mcan \pm SD) & $24.4 \pm 2.6$ & $26.6 \pm 4.0$ & $24.6 \pm 2.8$ \\
Old MI (n) & 15 & 0 & 15 \\
Post PCI (n) & 3 & 1 & 4 \\
Evaluation of chest pain (n) & 6 & 2 & 8
\end{tabular}

BMI, Body mass index; MI, Myocardial infarction; $\mathrm{PCl}$, Percutaneous coronary intervention

Table 2: Summary of $L V$ parameters measured by GSMPI and GSBPI

\begin{tabular}{|c|c|c|c|c|c|c|}
\hline LV parameters & & GSMPI & & & GSBPI & \\
\hline (MeantSD) & Male & Femalc & $\begin{array}{l}\text { All } \\
\text { patients }\end{array}$ & Malc & Femalc & $\begin{array}{l}\text { All } \\
\text { patients }\end{array}$ \\
\hline LVEF & $55.1 \pm 24.5$ & $71.3 \pm 29.7$ & $56.9 \pm 25.0$ & $53.1 \pm 24.6$ & $68.3 \pm 32.6$ & $54.8 \pm 25.3$ \\
\hline EDV & $98.6 \pm 55.3$ & $75.0 \pm 57.2$ & $95.9 \pm 54.9$ & $109.9=46.1$ & $105.7 \pm 66.1$ & $109.5=47.1$ \\
\hline ESV & $55.9 \pm 53.3$ & $38.0 \pm 58.0$ & $53.9 \pm 52.9$ & $59.7 \pm 50.8$ & $45.3 \pm 63.0$ & $58.1 \pm 51.1$ \\
\hline $\begin{array}{l}\text { Summed } \\
\text { perfusion score }\end{array}$ & $12.1 \pm 11.4$ & $5.7=8.9$ & $11.4 \pm 11.2$ & - & - & - \\
\hline $\begin{array}{l}\text { Summed } \\
\text { motion score }\end{array}$ & $22.9 \pm 17.0$ & $14.7 \pm 15.5$ & $21.9 \pm 16,8$ & - & - & - \\
\hline
\end{tabular}

LVEF, Left ventricular ejection fraction; EDV, End diastolic volume; ESV, End systolic volume.
A comparison of estimates of LVEF were between two methods at different ranges of LVEF, EDV, ESV, infarct size and RWMA is further summarized in Table 3.

Table 3: Comparison of LVEF between two methods at different ranges of LVEF EDV, ESV, infarct size and RWMA

\begin{tabular}{|c|c|c|c|}
\hline Study patients & $\begin{array}{c}\text { LVEF GSYIPI } \\
\text { Mean } \pm \text { SD }\end{array}$ & $\begin{array}{c}\text { LVEF GSBPI } \\
\text { Mean } \pm \text { SD }\end{array}$ & $\begin{array}{l}\text { Difference } \\
\text { Mean } \pm \text { SD }\end{array}$ \\
\hline LVEF $55 \%$ and above $(n=14)$ & $78.1 \pm 12.2$ & $76.2 \pm 9.7$ & $1.9 \pm 8.9$ \\
\hline LVEF below 55 to $35 \%$ ( $\mathrm{n}=7$ ) & $41.7 \pm 6.3$ & $40 \pm 12.9$ & $1.7 \pm 14$ \\
\hline LVEF below $35 \%(\mathrm{n}=6)$ & $25 \pm 3$ & $22 \pm 3.6$ & $3=5.9$ \\
\hline $\mathrm{EDV}<85 \mathrm{ml}(\mathrm{n}=16)$ & $74.3 \pm 15.7$ & $72.6 \pm 13.9$ & $1.8 \pm 10.3$ \\
\hline $85 \mathrm{ml}<\mathrm{EDV}<130 \mathrm{ml}(\mathrm{n}=3)$ & $39.7 \pm 5$ & $32.3 \pm 7.5$ & $7.3 \pm 9.5$ \\
\hline$E D V>130 \mathrm{ml}(\mathrm{n}=8)$ & $28.5 \pm 7$ & $27.6 \pm 13$ & $0.9 \pm 8.9$ \\
\hline $\mathrm{ESV}<15 \mathrm{ml}(\mathrm{n}=9)$ & $85.6 \pm 7.7$ & $81.2 \pm 6.5$ & $4.3 \pm 8.2$ \\
\hline $15 \mathrm{ml}<\mathrm{ESV}<60 \mathrm{ml}(\mathrm{n}=8)$ & $58 \pm 10.7$ & $57.3 \pm 16.9$ & $0.8 \pm 13.2$ \\
\hline $\mathrm{ESV}>60 \mathrm{ml}(\mathrm{n}=10)$ & $30.2 \pm 7.2$ & $29 \pm 12.2$ & $1.2 \pm 8.1$ \\
\hline No LV infarct $(\mathrm{n}=13)$ & $79.5 \pm 11.6$ & $77.3 \pm 9.1$ & $2.2 \pm 9.2$ \\
\hline Small LV infarct $(n=8)$ & $38.9 \pm 13.9$ & $35 \pm 16.2$ & $3.8 \pm 10.9$ \\
\hline Large LV infarct $(n=6)$ & $31.8 \pm 7.6$ & $32.3 \pm 14.8$ & $0.5 \pm 9.9$ \\
\hline No RWMA (n=10) & $83.6 \pm 9.7$ & $79.9 \pm 8.2$ & $3.7 \pm 9.9$ \\
\hline Mild to moderate RWMA $(\mathrm{n}=6)$ & $59.5 \pm 9.1$ & $55.5 \pm 18.8$ & $4=10.6$ \\
\hline Gross RIVMA (n=11) & $31.2 \pm 7.6$ & $31.6 \pm 14.4$ & $0.4 \pm 9.3$ \\
\hline
\end{tabular}

LVEF, Left ventricular ejection fraction; EDV, End diastolic volume; ESV, End systolic volume; RWMA, regional wall motion abnormality

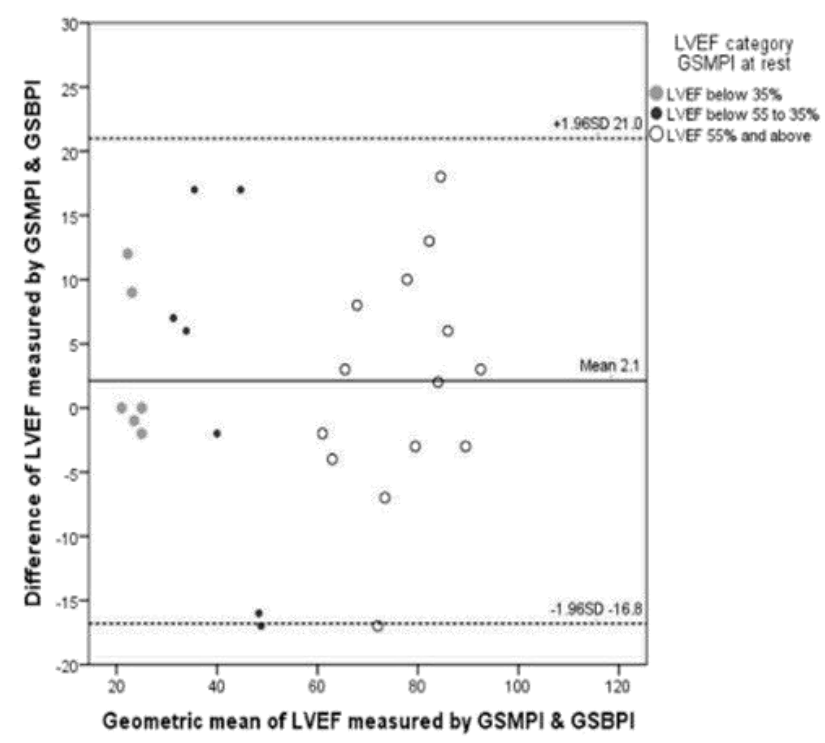

Figure 1(a) 


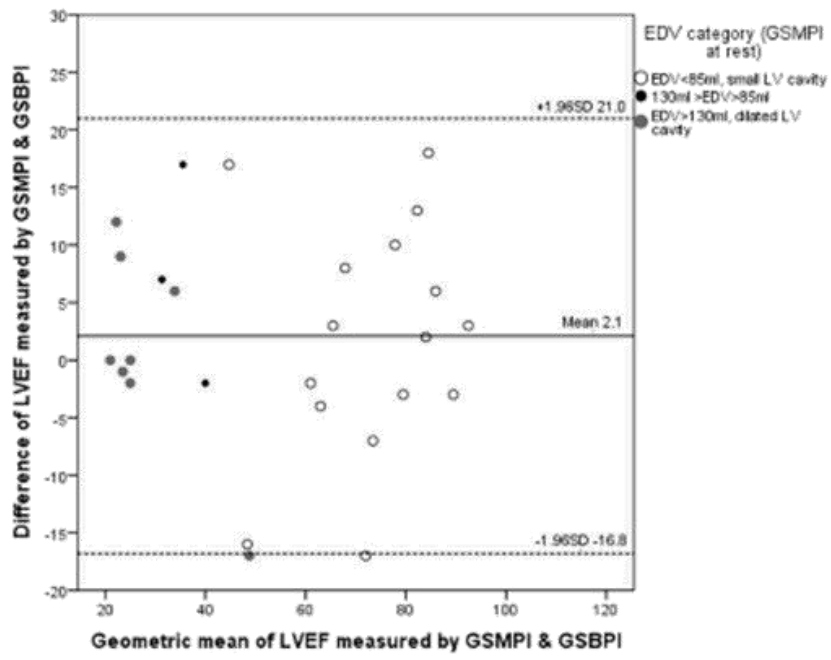

Figure 1(b)

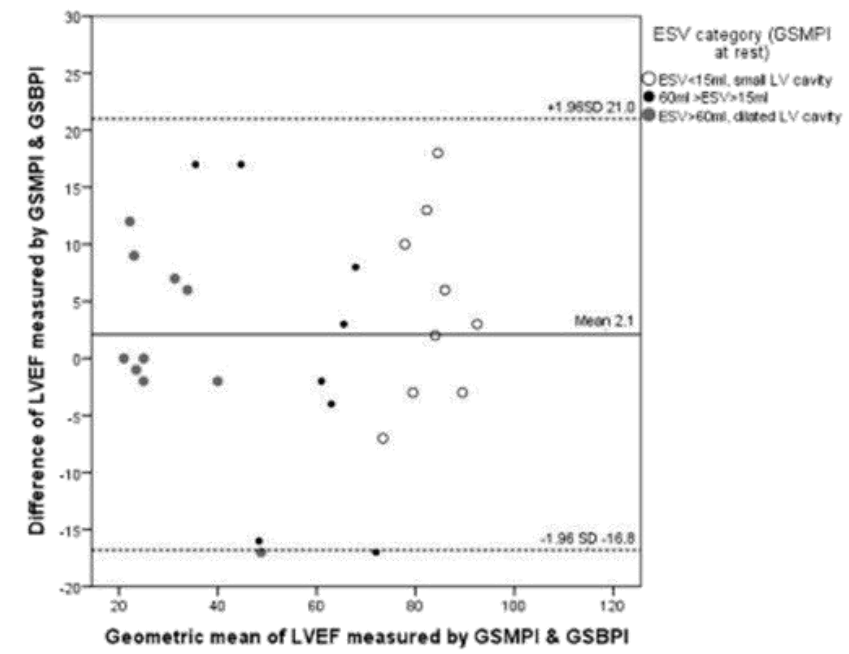

Figure 1(c)

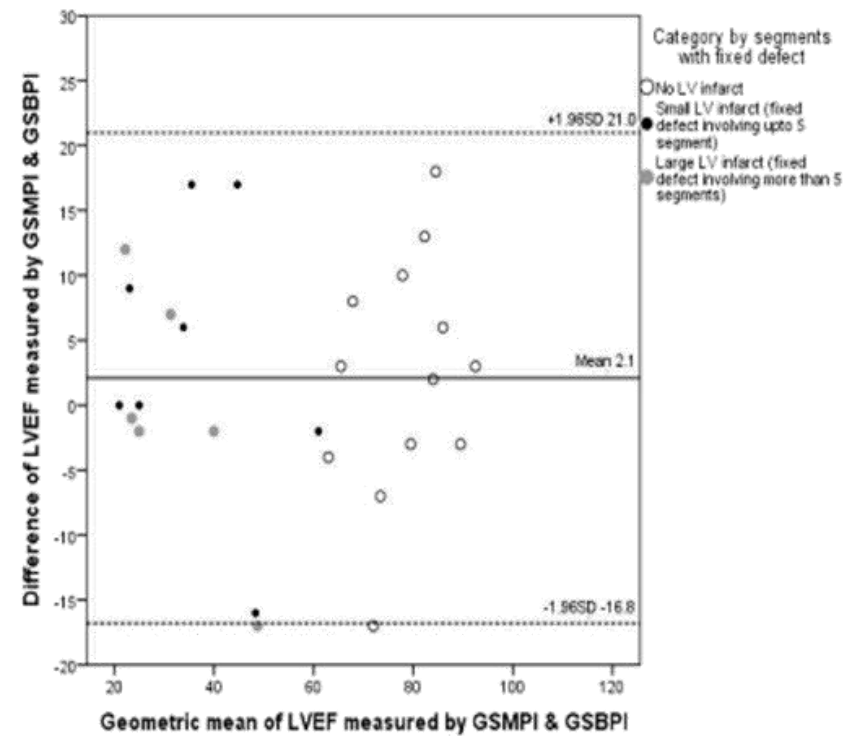

Figure 1(d)

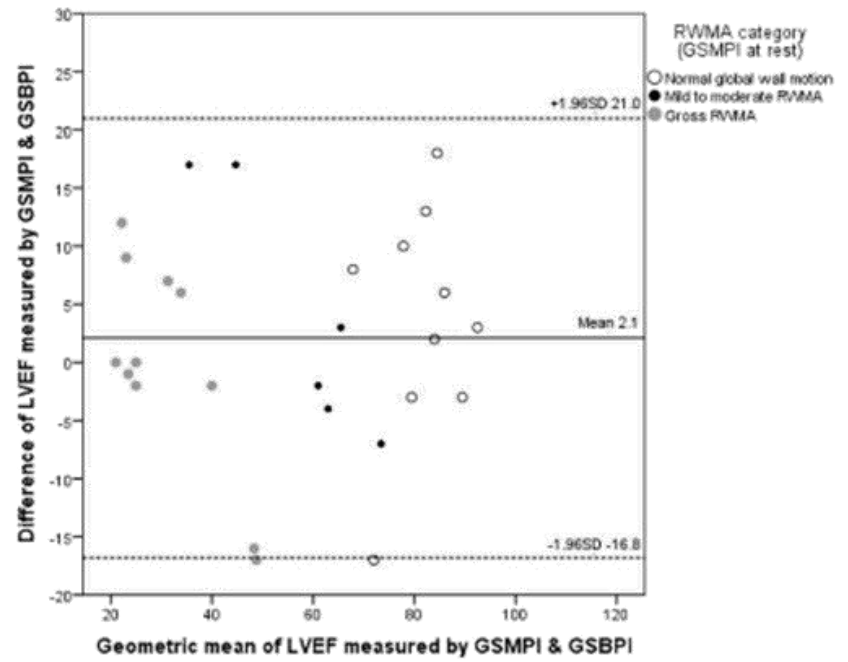

Figure 1(e)

Figure 1: Bland Altman plot showing agreement between GSMPI and GSBPI for measurement of LVEF at rest among ranges of LVEF (a), EDV (b), ESV (c), LV infarct size (d), RWMA (e) based on GSMPI results. Geometric means of LVEF measurements by GSMPI and GSBPI both at rest in each patient are plotted in $\mathrm{X}$ axis and difference between LVEF measurements by GSMPI and GSBPI both at rest in each patient are plotted in $\mathrm{Y}$ axis. The central line represents mean difference of LVEF measurements by two radionuclide methods. The outer pair of lines represents \pm 1.96 SD of difference.

\section{DISCUSSION}

The current study with Bland-Altman plots show that there was no significant difference between GSMPI and GSBPI for measurement of LVEF at rest in 27 patients with $\mathrm{CAD}$ i.e. the agreement between GSMPI and GSBPI for measurement of LVEF were significant. This finding was in concordance with findings by Paul et al. who reports no significant difference in the mean difference from zero for measurement of LVEF by GSMPI and GSBPI, both done with a frame rate of 10 per cardiac cycle (22). In addition Paul et al. in their study demonstrates underestimation of LVEF by GSMPI in comparison to GSBPI with a mean difference of $2.3( \pm 5.1)$. On the 
contrary current study showed slight overestimation of LVEF at rest by GSMPI in comparison to GSBPI with an overall mean difference of $2.1( \pm 9.6)$. In some recent case series similar overestimation of LVEF by GSMPI is claimed relative to planar blood pool radionuclide techniques $(17,19,23,24)$. While on contrary some groups report underestimation of LVEF by GSMPI compared with that by gated planar blood pool radionuclide techniques $(7,20,25,26)$.

All of these investigators who report underestimation of LVEF by GSMPI had used lower frame rates in GSMPI (eight frames/cardiac cycle) in comparison to blood pool techniques $(16,18 \& 24$ frames). However, Kumita et al. (25) who report an underestimation of LVEF by GSMPI had used frame rate of 32 for GSMPI and 25 frames/cardiac cycle for ERNV. All of the investigators who report overestimation of LVEF by GSMPI had used a gating of eight frames per cardiac cycle for GSMPI and higher frame rates (16 to 24) for blood pool technique. The current study too used a lower frame rate (eight frames/cardiac cycle) for GSMPI in comparison to GSBPI (16 frames/cardiac cycle) and discovered an underestimation of LVEF by GSMPI in comparison to GSBPI. Thus the postulated explanation of temporal under sampling of LV volumes in lower frame rate which has been suggested as a reason of underestimation of LVEF by gated radionuclide techniques may not stand true. In this study, agreement was also assessed after categorization of patients on basis of different ranges of LVEF, EDV, ESV, infarct size and wall motion. The agreement was however significant in all ranges.

The small sample size reduces the weight of inferences from this study. The sample size was small due to facts like interrupted supply of technetium generator, limited availability of pyrophosphate kit and busy patient schedule. This study, first in Bangladesh was attempted to assess if GSMPI agrees with gold standard GSBPI for measurement of LVEF. The result also shows an overall agreement between 4DMSPECT and QBS software for measurement of LVEF that matched with some earlier studies done using planar blood pool technique and QGS. Finally, results from this study may add confidence to put LVEF on MPI reports in most of the cases. Fact regarding frame rate may raises quest for explanations of underlying mechanisms. Need for determination of reference ranges and normal limit for SPECT measurement of LVEF, EDV, ESV, infarct size and RWMA is also warranted through larger trials in future.

\section{DISCLOSURE}

No competing financial interests exist

\section{ACKNOWLEDGEMENT}

Authors are indebted to Prof. M. Mozammel Haque, Chairman, Biochemistry Department of BSMMU who had been an indispensable guide to choose and adopt an appropriate statistical method in this study.

\section{REFERENCES}

1. Ghaffar A, Redidy KS, Singhi M. Burden of non-communicable diseases in South Asia. BMJ 2004;328(7443):807-10.

2. World Health Organization (WHO) Regional Office for South-East Asia (SEARO). Health Situation in the South-East Asia Region 2001-2007. WHO Regional Publication, South-East Asia Series No. 46. 2008; WHO/SEARO, New Delhi.

3. World IIcalth Organization - NCD Country Profiles, 2011 Bangladesh, fact shect, vicwed 19 November 2016 , http://www.who.int/nmh/countries/bgd_en.pdf

4. Hachamovitch R, Berman DS, Shaw LJ, Kiat H, Cohen I, Cabico $\mathrm{JA}$, et al. Incremental prognostic value of Myocardial perfusion single photon emission computed tomography for prediction of cardiac death: differential stratification for the risk of cardiac death and myocardial infarction. Circulation 1998:97(6):535-43.

5. Sharir T, Germano G, Kavanagh PB, Lai S, Cohen I, Lewin HC et al. Incremental prognostic value of post-stress left ventricular cjection fraction and volume by gated myocardial perfusion single photon emission computed tomography. Circulation 1999;100(10):1035-42.

6. Bavelaar-Croon CD, Pauwels EK, van der Wall EE. Gated single-photon emission computed tomographic myocardial imaging: a new tool in clinical cardiology. Am Heart J 2001;141(3):383-90.

7. Mohan HK, Livieratos L, Gallagher S, Bailey DL, Chambers I, Fogelman, I. Comparison of myocardial gated single photon cmission computerized tomography, planar radionuclide ventriculography and cchocardiography in cvaluating lcft ventricular cjection fraction, wall thickening and wall motion. Int J Clin Pract 2004;58(12):1120-26. 
8. Groch MW, DePuey EG, Belzberg AC, Erwin WD, Kamran M, Barnett CA et al. Planar imaging versus gated blood-pool SPECT for the assessment of ventricular performance a multicenter study. f Nucl Med 2001;42(12);1773-9.

9. Nasreen F, Nisa L, Sarker AK, Kabir MF, Journey of Nuctear Cardiology in Bangladesh - A report, Bangladesh J Nucl Med $2009 ; 12(1): 108$

10. IIssse B, Lindhardt TB, Acampa W, Anagnostopoulos C, Ballinger J, Bax JJ ct al EANM/ESC guidelines for radionuclide imaging of eardiac function. Eur I Nuel Med Mol Imaging 2008:35(4):851-85.

11. Vieira1 S,CorrenteJE: Statistical methods for assessing agreement between double readings of clinical measurements. $\mathrm{J}$ Appl Oral Sci $2011 ; 19(5) ; 488-92$

12. Liao, JJ. Sample size calculation for an agreement study. Pharma Stat, 2010,9(2):125-32.

13. Klocke FJ, Baird MG, Lorell BH, Bateman TM, Messer JV, Berman DS et al. ACC/AIIA/ASNC guidelines for the clinical use of eardiac radionuclide imaging--executive summary: a report of the American College of Cardiology/American Heart Association Task Force on Practice Guidelines ( $\Lambda$ CC/NHA $/$ SNC Committee to Revise the 1995 Guidelines for the Clinical Use of Cardiac Radionuclide Imaging): Circulation 2003;108(11) 1404-18.

14. Kabìuzzamạn $\mathrm{M}$, Ali $\mathrm{MA}$, Isläm $\mathrm{MN}$ Coronary angiographic characteristics of patients with first myocardial înfarction admitted in a tertiary care cardiac hospital in Bangladesh. Cardiovase J $2010 \div 2(2): 204-11$

15. Abraham WT, Ilayes DL. Cardiac resynchronization therapy for heart failure. Circulation 2003; 108(21):2596-603.

16. Chuang ML, Hibberd MG, Salton CI, Beaudin RA, Ríley MF, Parker RA et al. Importance of imaging method over imaging modality in noninvasive determination of left ventricular volumes and ejection fraction assessment by two- and three-dimensional echocardiography and magnetic resonance iniging: $\mathrm{J}$ Am Coll Cardiol 2000;35(2):477-84.

17. Khali MM, Elgazzar A, Khatil W, Omar A, Ziada G. Assessment of left ventricular cjcetion fraction by four different methods using Tc-99mtetrofosmin gated SPECT in patients with small hearts: correlation with gated blood pool. Nuel Mcd Commun $2005,26(10): 885-93$.
18. Ford $\mathrm{PV}_{\text {, }}$ Chatzioannou. SN, Moore WH, Dhekne RD. Overestimation of the LVEF by Quantitative Gated SPECT in Simulated Lef Ventricles, J Nucl Med 2001;42(3):454-59.

19. Xia W, Ly Z, Wang G, Cai H, Ni J, Zhang Y el al A comparison and validation of blood-pool imaging and ECG-gated SPET myocardial perfision imaging to assess left ventricular ejection fraction: Hell J Nucl Med 2010;13(3):24T 45 .

20. Teresinska A, Whuk J, Konicezna S, Dabrowski A Verification of the left ventricular ejection fraction from gatod myocardial perfusion studies (GSPECT).Kardiol Pol 2005;63(5):465-77. (Abstract)

21. Nasreen $F$, Nisa $\mathrm{L}$, Hoque $\mathrm{H}$, Sarker AK. Evaluation of left ventricular ejection fraction and volumes in patients with dilated cardiomyopathy: Gated single photon emission compuled tomography versus two dimensional echocardiography, Bangladesh J Nüel Med 2012;15(1):54-7.

22. Paul AK, Hascgawa $\mathrm{S}$, Yoshioka J, Yamaguchi H, Tsujimura $\mathrm{E}$, Nishimura; T. Assessment of left ventricular function by gated myocardial perfusion and gated blood-pool SPECT can we use the same reference database? Ann Nucl Med 2000;14(2):75-80.

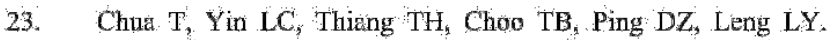
Accuracy of the automated assessment of left ventricular function with gated perfusion SPECT in the presence of perfusion defects and left ventricular dysfunction correlation with equilibrium radionuclide veniriculography and echocardiography. I Nucl Cardiol 2000; 7(4);301-11.

24. Godkar D, Bächu K, Dave B, Mcgnà R, Niranjan S, Khanna A. Comparison and co-relation of invasive and noninvasive methods of ejection fraction measurement. J Natl Med Assoc $2007 ; 99(11): 1227-28,1231-34$.

25. Kumita S, Choo K, Nakajo H, Toba M, Uwamori M, Mizumara S et al. Assessment of left ventricular diastolic function with electrocardiography-gated myocardial perfusion SPECT: comparison with multigated equilibrium radionuclide angiography, J Nucl Cardiol 2001,8(5); 568-74,

26. Canbaz $\mathrm{F}_{\text {; }}$ Basoglu $\mathrm{T}$, Semirgin $\mathrm{SU}_{3}$ Yapici $\mathrm{O}$ Yazici $\mathrm{M}_{*}$ Cold infarction arcass of varying size in the presence of left ventricular. dysfimetion: the impact on left ventricular cjection fraction determination by gated SPECT compared to radionuclide ventriculography Hell J of Nucl Med 2005;8(3) 149-53 (Abstract) 